\title{
Bronchoalveolares Karzinom: Bestrahlen!
}

Das bronchoalveoläre Karzinom (BAK) gehört zu den milderen Subtypen des nichtkleinzelligen Lungenkarzinoms (NSCLC). Vielleicht wird das BAK deshalb so selten bestrahlt, wenn es nicht resezierbar ist. Aber wäre die Radiotherapie nicht ebenso erfolgversprechend wie beim NSCLC?

— rüher wurde zwischen kleinzelligen oder nichtkleinzelligen Lungenkarzinomen unterschieden. Zunehmend wird aber erkannt, wie bedeutend die weitere Differenzierung beim NSCLC ist. Das $\mathrm{BAK}$, zu den Adenokarzinomen gehörig, repräsentiert 4-24\% der NSCLC-Fälle und hat tendenziell eine gute Prognose. In der Therapie des BAK aber ist vieles ungeklärt. Während Patienten mit nicht resezierbarem NSCLC gemeinhin eine Radiotherapie erhalten, gab es bisher quasi keine Studien zum Wert der Radiotherapie beim BAK. In den Leitlinien des American College of Chest Physicians ist das Wort Radiotherapie nicht einmal erwähnt. Nun liegt eine populationsbasierte Studie vor, in die Daten von 6.933 zwischen 2001 und 2007 behandelten Patienten mit BAK im Stadium I-III eingingen. Das mediane Alter zum Zeitpunkt der Diagnose betrug 70 Jahre (10101 Jahre). Die Mehrzahl der Patienten $(74,4 \%)$ befand sich in Stadium I; $968 \mathrm{~Pa}$ -

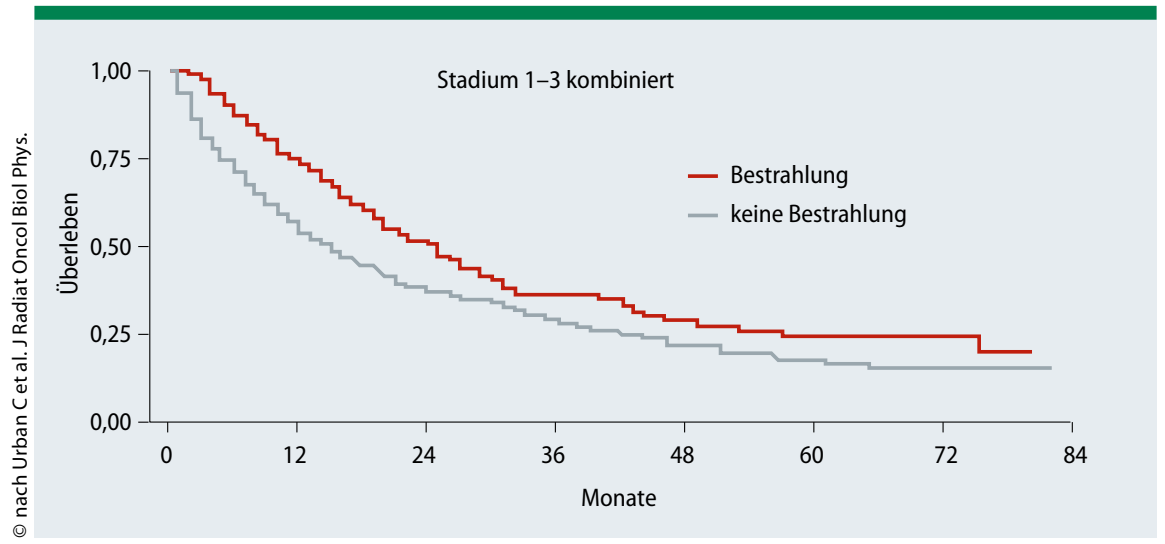

Abb.: Patienten mit BAK profitieren in allen Stadien von einer Bestrahlung. tienten (14\%) wurden nicht operiert. Patienten ohne Resektion waren tendenziell älter ( $\mathrm{p}<0,0001)$, häufiger männlich $(\mathrm{p}=0,001)$, von schwarzer Hautfarbe ( $\mathrm{p}$ $<0,0001)$ und befanden sich häufiger im Stadium III der Erkrankung ( $p<0,0001)$. Ihr geschätztes 2-Jahres-Gesamtüberleben betrug in den Stadien I, II bzw. III 58, 44 bzw. $27 \%$.

Von den Patienten ohne Resektion wurden nur 300 (31\%) bestrahlt. Dabei gingen mit einem besseren Überleben weibliches Geschlecht, ein früheres Krankheitsstadium zum Zeitpunkt der Diagnose einher - und Bestrahlung: Das mediane Überleben von Patienten ohne vs. mit Radiotherapie betrug im Stadium I $(\mathrm{n}=364) 28$ vs. 33 Monate $(\mathrm{p}=0,06)$; im Stadium II $(\mathrm{n}=3) 18$ Monate vs. nicht erreicht und im Stadium III $(\mathrm{n}=517) 10$ vs. 17 Monate $(\mathrm{p}=0,003)$.

Fazit: Eine Radiotherapie ist mit einer verbesserten Prognose des nicht resezierten BAK im Stadium I-III verbunden. Allerdings erhielt weniger als ein Drittel der Patienten, die potenziell von einer Radiotherapie hätten profitieren können, eine solche. Dies lässt vermuten, dass Ärzte, die in die Behandlung dieser Patienten involviert sind, die Bedeutung der Radiotherapie zu gering einschätzen.

Christina Berndt

Urban C et al. Radiotherapy improves survival in unresected stage I-III bronchoalveolar carcinoma. Int J Radiat Oncol Biol Phys. 2012;84(3):780-5. 